\title{
Biopsy of breast cancer metastases: patient characteristics and survival
}

\author{
Shlomit Strulov Shachar ${ }^{1}$, Tanya Mashiach², Georgeta Fried ${ }^{1}$, Karen Drumea ${ }^{1}$, Noa Shafrann ${ }^{4}$, Hyman B. Muss ${ }^{3}$ \\ and Gil Bar-Sela ${ }^{1,4,5^{*}}$
}

\begin{abstract}
Background: Discordance in hormone receptors (HR) and human epidermal growth factor receptor 2 (HER2) status between primary tumors and metastatic sites for breast cancer is well established. However, it is uncertain which patient-related factors lead to biopsy when metastases are suspected and whether having a biopsy impacts survival.

Methods: The medical charts of metastatic breast cancer (MBC) patients diagnosed January 2000-August 2014 were retrospectively reviewed. A biopsy was defined as a procedure where tissue was obtained and assessed for both HR and HER2. Both bivariate and multivariate analyses were performed to assess patient characteristics related to biopsy and whether having a biopsy was associated with improved survival.

Results: Of 409 patients suspected of having MBC, 165 (40\%) had a biopsy, and 34\% of these had discordant HR or HER2 status when compared to the initial diagnosis. In multivariate analysis, having a biopsy was associated with: recurrence in years 2010-2014, disease-free interval of $>=3$ years, stage 0-IIA at presentation, suspected locoregional recurrence, being HR+/HER2-, or missing HR/HER2 at diagnosis. A similar multivariate analysis revealed that having a biopsy was associated with improved survival ( $H R=0.67, p=0.002)$. The association of biopsy and improved survival was noted in specific subgroups: patients with missing HR and HER2 data at initial diagnosis $(p=0.001)$, those without metastases in liver, lung or brain ( $p=0.001)$, and being younger than 70 years old at recurrence $(p<0.001)$.
\end{abstract}

Conclusions: Specific clinical factors were associated with biopsy at the time of suspected recurrence. Having a biopsy was associated with reduced mortality.

Keywords: Biopsy, Breast carcinoma, Pathology, Recurrent-metastatic disease, Survival

\section{Background}

Breast cancer is the most common cancer among women in the United States, and it is estimated that 246,660 new cancer cases will be diagnosed in 2016 [1]. Despite recent progress in diagnostic and therapeutic approaches of early breast cancer, many patients develop metastases, resulting in about 40,000 deaths annually. Systemic treatment in metastatic breast cancer (MBC), as in early stage breast cancer, is chosen on the basis of estrogen receptor (ER) and progesterone receptor (PR) status and overexpression/amplification of the human epidermal growth factor receptor 2 (HER2). In addition,

\footnotetext{
* Correspondence: g_barsela@rambam.health.gov.il

'Division of Oncology, Rambam Health Care Campus, Haifa, Israel

${ }^{4}$ Rappaport Faculty of Medicine, Technion-Israel Institute of Technology, Haifa, Israel

Full list of author information is available at the end of the article
}

other factors are routinely used for therapy decisions, such as disease-free interval, site(s) of relapse, and number of metastases $[2,3]$.

Discordance in hormone receptors and HER2 status between the primary tumor and the metastatic lesion is well documented [4]. A recent review demonstrated a large range of receptor discordance with ER, PR and HER2 discordance of $6-40 \%, 21-41 \%$ and $1-43 \%$, respectively [5]. In addition, a meta-analysis of 48 studies, in which 4200, 2739, and 2987 tumors were evaluated for ER, PR and HER2 discordance, respectively, showed a discordance range of $8-23 \%$ [6]. The reasons for discordance may relate to changes in tumor biology between the initial tumor and the metastatic lesion biopsied, heterogeneity within the tumor itself, and/or imperfect accuracy and reproducibility of assays, although the 
relative contribution of each of these factors to the overall discordance rate is unclear. The unequivocal recommendations for biopsy when metastases are suspected are: solitary metastasis, unusual clinical course, and research [7]. The existent discordance between the primary tumor and recurrent metastasis in ER, PR and HER2 status in portions of tumors has been demonstrated in many studies $[5,8]$.

The Advanced Breast Cancer (ABC) consensus guidelines recommend biopsying a metastatic lesion, if easily accessible, and confirming the diagnosis, particularly when metastasis is diagnosed for the first time [3]. The National Comprehensive Cancer Network (NCCN) Panel recommended doing a biopsy to confirm recurrence, and repeating ER/PR and HER2 at the metastatic site [2].

Biopsy results may also be related to survival. In a recent Swedish trial, it was concluded that patients with tumor loss of ER or PR during progression have worse survival than patients with retained receptor expression [8]. However, in another retrospective trial, no significant survival difference between patients with discordant versus nondiscordant receptors in MBC was seen [9]. Lastly, Curtit et al. reported that patients who had undergone biopsy had survival benefit (median OS 79 months vs 32 months, $p=0.0001)$ [10].

However, it is still unclear whether patient characteristics are associated with having a biopsy and whether having a biopsy is associated with improved survival. Since prospective trials to address these questions are not likely to be performed, we conducted a large retrospective trial to help answer these questions.

\section{Methods}

\section{Study design}

After approval of the study protocol by the institutional ethics committee - Helsinki Committee of Rambam Health Care Campus (RHCC) (Certificate no. 0408-13$\mathrm{RMB}$ ) - a retrospective analysis was conducted of all medical records of adult patients treated in the Division of Oncology at RHCC in Haifa, Israel, for metastatic breast carcinoma from January 2000 to August 2014. Eligibility for the study included having metastatic/locoregional non-curable disease, previous treatment for early breast cancer with curative intent (including surgery), and an interval of at least 6 months since the end of adjuvant chemotherapy, radiotherapy, and/or surgery (adjuvant hormonal therapy was allowed). Excluded from the study were patients with de novo primary metastatic disease and patients who were not treated with curative intent at first diagnosis (without surgery). Patients were de-identified after data were collected from digital and non-digital records. Data extracted from the medical files included demographics and medical history: age, marital status, number of children, stage (American Joint Committee on Cancer, $7^{\text {th }}$ edition), grade, ER, PR and HER2 status (from initial surgery/biopsy specimen), adjuvant treatment regimen, and type of surgery at initial diagnosis. Data obtained on patients with suspected metastases included the sites of metastasis, if and when a biopsy was done, a pathology report from the biopsy including documentation of metastatic adenocarcinoma, ER, PR and HER2status, and date of death or date of last follow-up. We defined a biopsy as a procedure confirming adenocarcinoma, including reassessment of HR and HER2 status. Patients were defined as not having a biopsy if they did not undergo biopsy, had a biopsy which only histologically confirmed metastases, or did not reassess HR and HER2 status. Positive ER or PR expression was defined as $1 \%$ of cells or greater staining for ER or PR. HER2 assessment was scored from $0-3+$. In case of overexpression scored at $2+$, fluorescent in situ hybridization (FISH) was performed. HER2 was considered positive if overexpression was scored at $3+$ in immunohistochemistry, or $2+$ in immunohistochemistry, if FISH was positive.

\section{Statistical analysis}

Logistic regression was used to calculate the odds ratios (OR) with 95\% confidence intervals (95\% CI) and $p$ values in bivariate analysis to determine associations between tumor characteristics and likelihood of biopsy. Based on previous theory [11], variables with $p<=0.1$ were included in subsequent Multivariable Forward Stepwise Logistic Regression analysis. The HosmerLemeshow goodness-of-fit statistic [11] was calculated, and the area under the receiver operating characteristic (ROC) curve was used as a measure of model discrimination. Bivariate Cox regression was then used to calculate hazard ratios (HR) with 95\% confidence intervals (CI) and $p$ values for overall survival (OS). Stratified analysis was used to assess confounding. Variables with $p<=0.1$ were used in multivariate Cox Regression analysis to assess the effect of biopsy on overall survival (OS), as well as the effect of patient and tumor characteristics. Two-tailed $p$ values of 0.05 or less were considered statistically significant. Statistical analyses was performed using SPSS (Statistics Products Solutions Services) 21.0 software for Windows.

\section{Results}

\section{Patient characteristics}

The medical records of 409 patients diagnosed with MBC between 2000 and 2014 were evaluated. Of these 409 patients, $165(40 \%)$ patients had a biopsy as defined in this study, while 90 (22\%) had only histologic confirmation of metastases.

Median follow-up for metastatic disease was 30.2 months (range, 1.1-154.2 months) for all patients and 42.3 months (range, 1.3-155.8 months) for patients who were alive at 
the time of chart review. Patient characteristics are shown in Table 1. Median age at metastatic diagnosis was 57.4 years (range, 26.6-88.7 years). Of the 409 patients, $42 \%$ were initially diagnosed with stage 0-IIA tumors and $57 \%$ with stage IIB-III tumors. Also at initial diagnosis, 186 (45\%) patients had grade 1-2 tumors, 146 (36\%) had grade 3 tumors (19\% data missing), 282 (69\%) were HR positive and 92 (22\%) were HER2 positive.

All patients underwent surgery with curative intent. More than $75 \%$ of the patients received chemotherapy (72\% anthracycline-based), either as adjuvant or neoadjuvant treatment. Adjuvant trastuzumab was given to only $8 \%$ of patients. Adjuvant hormonal therapy was given to $62 \%$ of patients. Biopsy sites were: locoregional recurrence (skin, chest wall, breast or lymph nodes (LN)) 77 (47\%), bone 33 (20\%), liver 26 (16\%), lung 14 (8\%), abdomen 5, other sites 10 .

\section{Clinical characteristics associated with biopsy}

Clinical characteristics found to be significantly associated with having a biopsy are shown in Table 1 . Overall, the percentage of patients who underwent biopsy significantly increased between 2000 and 2014. Factors related to having a biopsy included: not having HR or HER 2 status at initial diagnosis $(\mathrm{OR}=5.96, p<0.0001)$, disease-free interval longer than $>=3$ years $(\mathrm{OR}=5.42, p<0.0001)$, and locally advanced disease (in the breast, LN, skin, or chest wall $)(\mathrm{OR}=3.06, p<0.0001)$. Advanced stages IIB-III $(\mathrm{OR}=0.40, p<0.0001)$ and high grade (grade 3 compared to grade $1(\mathrm{OR}=0.28, p=0.026))$ were associated with fewer biopsies. Of note, patient age at time of suspected metastases was not associated with the biopsy decision.

In multivariate analysis (Table 2), variables that remained significantly associated with biopsy were: year of recurrence $(\mathrm{OR}=10.67, \quad p<0.0001$ for 2010-2014), disease-free interval of $>=3$ years $(\mathrm{OR}=3.20, p<0.001)$, stage 0 -IIA ( $\mathrm{OR}=1.89, p=0.012$ ), locally advanced disease $(\mathrm{OR}=1.90, p=0.011), \mathrm{HR}+\mathrm{HER} 2-$ at initial diagnosis $(\mathrm{OR}=2.83, p=0.021)$, and unknown HR or HER2 at initial diagnosis $(\mathrm{OR}=6.78, p<0.001)$.

\section{Discordance in hormone receptor and HER2 status among primary and metastatic tumors}

In 179 patients whose tumor HR status was evaluated both at primary diagnosis and upon recurrence, HR status changed from positive to negative in 27 (15\%) patients, and from negative to positive in 14 (8\%) patients. HER2 status was evaluated in 114 patients at both diagnosis and recurrence. HER2 changed from positive to negative in 5 (4\%) patients, and from negative to positive in $10(9 \%)$ patients. Figure 1 describes changes from initial pathology to biopsy in 107 patients whose HR and HER2 were measured in both biopsies.

\section{Factors associated with overall mortality}

In bivariate analysis, clinical characteristics associated with overall mortality are summarized in Table 1. At diagnosis, advanced stage (IIB, III), higher grade (grade 3 ), and having a triple negative or HR-/HER2+ tumor was associated with worse survival. In turn, at the time of metastases, age $>=70$ years, a disease-free interval of $<3$ years, multiple metastatic sites, brain, liver and lung metastases, or having triple negative disease was associated with worse survival. Of note, having a biopsy upon recurrence was associated with longer survival $(\mathrm{HR}=0.64, p<0.0001)$.

Multivariate analysis of factors associated with shortened overall survival at initial diagnosis (Table 3 ) included advanced stage (stage IIB-III) $(\mathrm{HR}=1.40, p=0.004)$, triple negative phenotype $(\mathrm{HR}=2.14, p=0.001)$, and $\mathrm{HR}$ $+/ \mathrm{HER} 2$ - phenotype $(\mathrm{HR}=1.61, p=0.013)$. At the time of metastases, age $\geq 70$ years $(\mathrm{HR}=1.65, p=0.002)$, and brain or liver and lung metastasis $(\mathrm{HR}=2.85, \mathrm{HR}=2.46$, respectively, $p<0.001)$ were associated with shortened overall survival. As in bivariate analysis, not having a biopsy was associated with shorter survival as compared to having a biopsy $(\mathrm{HR}=0.67, p=0.002)$.

\section{Association of overall survival and biopsy in selected subgroups}

Exploratory analysis of the interaction of biopsy and survival was performed in selected subgroups (Table 4). A significant survival benefit was found in patients with missing data on receptor status $(p=0.001)$ at the initial diagnosis, no metastasis in liver and lung or brain $(p=$ $0.001)$, or younger than age $70(p=0.0001)$. There was no significant effect on survival among different tumor phenotypes; however, this might be due to the small number of patients in each group.

\section{Discussion}

The primary purposes in doing a biopsy when $\mathrm{MBC}$ is suspected are to confirm the diagnosis of metastasis and to reassess HR and HER2 status, two key factors that influence subsequent treatment. In a prospective trial that included 64 women with a history of prior breast cancer and suspected new metastases, a non-breast cancer diagnosis was found in 14, and $10 \%$ had benign findings [12]. Of the 205 patients prospectively included in the BRITS trial, 9\% did not have recurrent disease on biopsy [13]. In our study, $33 \%$ of patients had a biopsy only for histological confirmation of $\mathrm{MBC}$. We also found that, in recent years, physicians ordered significantly more biopsies $(\mathrm{OR}=10.67, p<0.0001$ for 2010-2014), perhaps due to the fact that recent studies have emphasized that receptor status can change [10, 14].

The main purpose of a biopsy is to reassess the HR and HER2 status of the metastatic disease. In our study, 


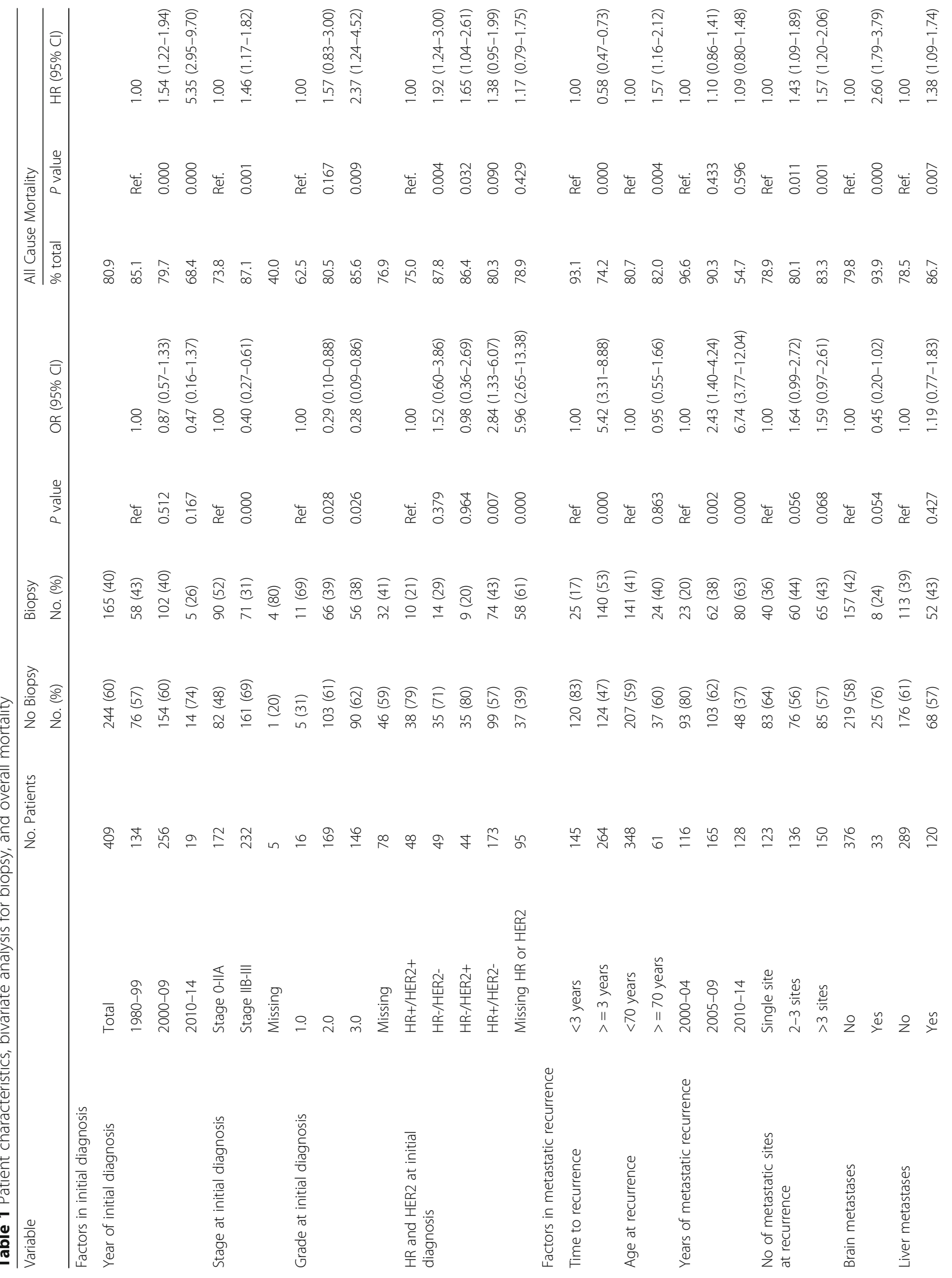




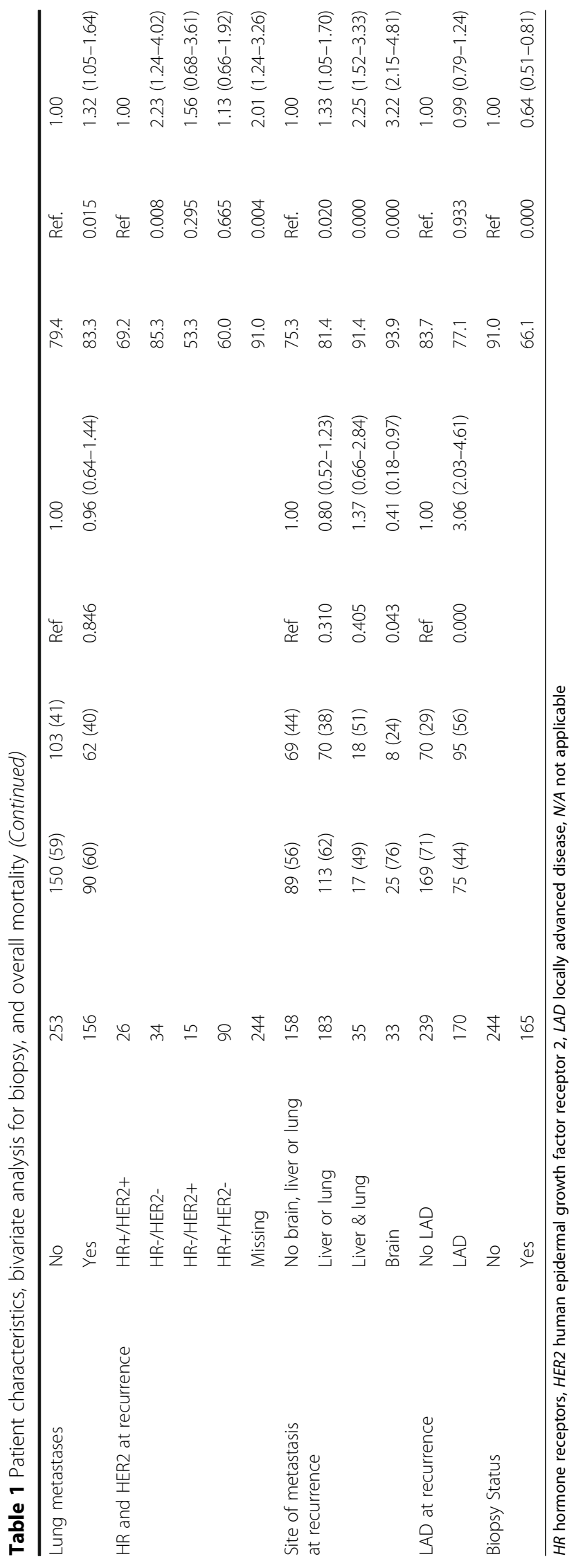


Table 2 Multivariate analysis : Factors predictive of biopsy

\begin{tabular}{llll}
\hline Variable & & $P$ value & Adjusted OR (95.0\% Cl for OR) \\
\hline Years of metastatic recurrence & $2000-04$ & & Ref. \\
& $2005-09$ & .000 & $3.79(1.94-7.40)$ \\
Time to recurrence(years) & $2010-14$ & .000 & $10.67(5.13-22.22)$ \\
& $<3$ years & Ref. & $3.20(1.78-5.76)$ \\
Stage at initial diagnosis & $>=3$ years & .000 & Ref. \\
Site of metastasis at recurrence & Stage IIB-III & & $1.89(1.15-3.10)$ \\
& Stage 0- IIA & Ref. \\
HR \&HER2 at initial diagnosis & No locally advanced disease & .012 & $1.90(1.16-3.10)$ \\
& Locally advanced disease & & Ref. \\
& HR+/HER2+ & .011 & $1.68(0.57-4.93)$ \\
& HR-/HER2- & .349 & $1.15(0.35-3.74)$ \\
\hline
\end{tabular}

All other variables were not significant

$H R$ hormone receptors, HER2 human epidermal growth factor receptor 2

a biopsy that included confirmation of HR and HER2 status was associated with a significant survival benefit $(p=0.001)$. In a study by Amir et al. [14], there were discordant findings between findings at initial diagnosis and findings after metastasis in $37.6 \%$ of patients, resulting in a treatment change in $14 \%$ of patients. However, no change in OS was noted between concordant and discordant groups in that study [14]. Different results were seen in a study in China, which showed worse OS for patients who had receptors discordance in the biopsy [15]. However, in a review of 13 recent major studies regarding biopsy in $\mathrm{MBC}$, the impact of this procedure on survival was not clear [16].

Improved survival for patients whose metastases retained ER or PR expression as compared to those with loss of ER or PR expression was shown by Karlsson et al. in a cohort of 177 patients with MBC [8], and in a pooled analysis of two prospective studies (BRITS and DESTINY studies) that included 289 MBC patients, where a gain of HR or HER2 expression led to most changes in treatment approaches [17]. We demonstrated that a biopsy that included confirmation of HR and

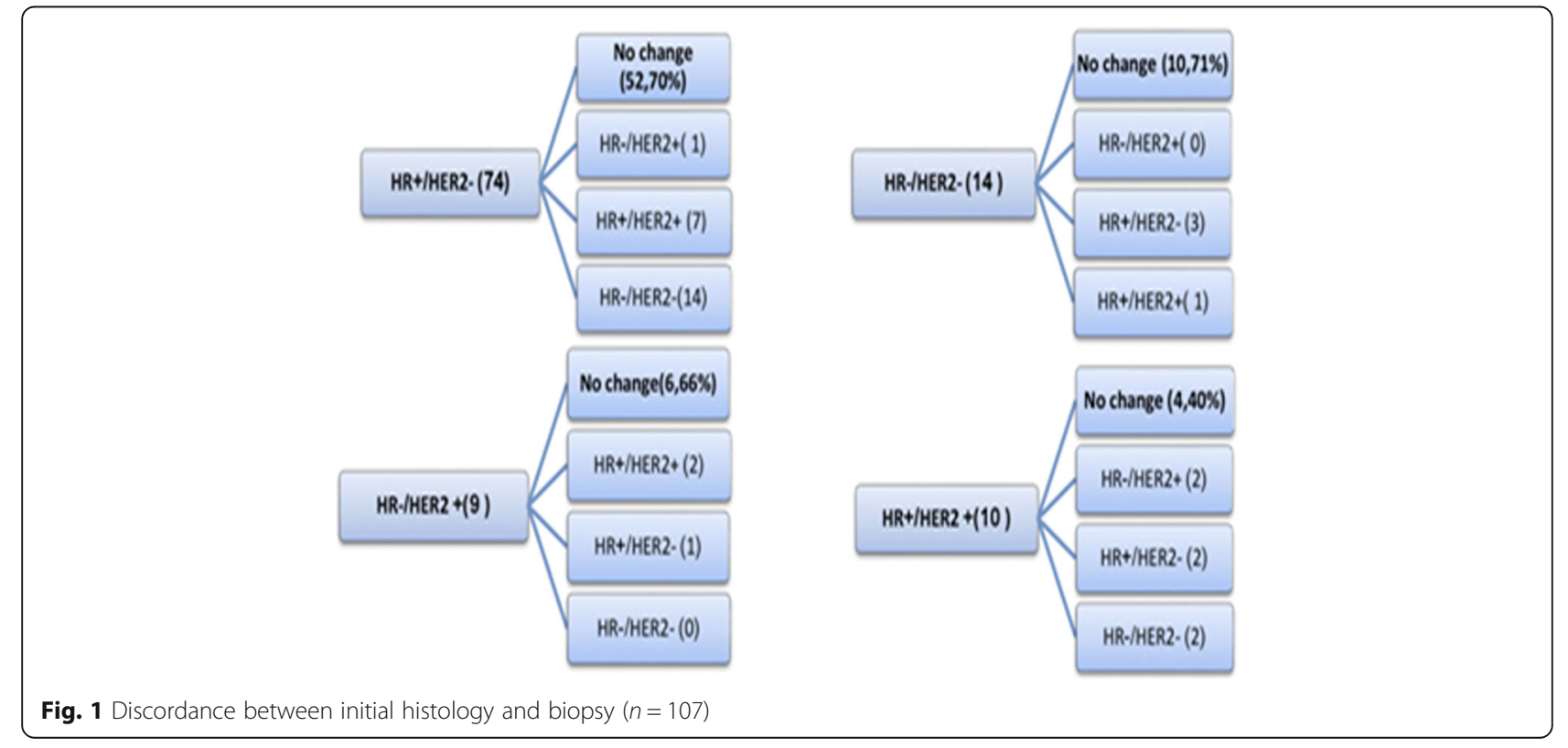


Table 3 Multivariate analysis: Factors predictive of All Cause mortality

\begin{tabular}{llll}
\hline Variable & & $P$ value & HR Adjusted(95.0\% Cl for HR) \\
\hline HR \& HER2 at initial diagnosis & HR+/HER2+ & Ref. & $2.14(1.36-3.39)$ \\
& HR-/HER2- & .002 & $1.36(0.86-2.27)$ \\
& HR-/HER2+ & .179 & $1.61(1.11-2.29$ \\
& HR+/HER2- & .013 & $1.49(0.98-2.21)$ \\
& HR and/or HER2 missing & .065 & Ref. \\
Site of metastasis at recurrence & No brain, liver or lung metastasis & & $2.46(1.68-3.60)$ \\
& Liver \& lung involvement & .000 & $2.85(1.88-4.31)$ \\
Age at metastatic recurrence (years) & Brain involvement & .000 & Ref. \\
Stage at initial diagnosis & $<70$ & & $1.64(1.19-2.24)$ \\
& $>=70$ & .002 & Ref. \\
Biopsy & Stage 0-IIA & & $1.40(1.12-1.76)$ \\
& Stage IIB-III & .004 & Ref. \\
\hline
\end{tabular}

All other variables were not significant

$H R$ hormone receptors, HER2 human epidermal growth factor receptor 2

HER2 was associated with lower mortality. However, the major benefit was seen mainly in patients who had missing data on HR or HER2 status at initial diagnosis $(p=0.001)$.

We offer some explanations for the association of undergoing biopsy and improved survival: biopsy may show discordance with initial findings that may be associated with improved treatment selection, in turn, resulting in a higher response rate, longer progression free interval, and possibly improved survival. This explanation is supported by recent data showing that molecular subtyping and gene modules of post-relapse biopsy are associated with survival [18]. In our study, $8 \%$ of patients who were initially HR- became $\mathrm{HR}+$, suggesting that endocrine therapy should be considered. At the same time, $15 \%$ of patients who were $\mathrm{HR}+$ at diagnosis became HR- at biopsy and would likely not have benefitted from endocrine therapy; for these patients, chemotherapy would be a better choice. In $9 \%$ of patients, HER2- changed to HER2+, a change that can modify the treatment plan to include anti-HER2 directed therapy; anti-HER2 directed therapies significantly improved survival for patients with HER2+ MBC [19, 20]. Of note, a small group, $4 \%$ of patients, changed from HER2+ to HER2- and most likely would not have benefitted from anti-HER2 therapy. Another possible explanation for what we observed in our sample is that physicians choose to biopsy patients whose clinical scenarios are associated with improved survival, such as stage at initial presentation and longer disease-free intervals (Table 2). To test this scenario, a prospective randomized control trial would be needed.

Although no difference was found in biopsy percentages by age groups, patients $<70$ years who had a biopsy were found to have improved survival in subgroup analysis $(p<0.0001)$. This finding might be partially due to competing causes of mortality in older patients and the fact that older patients are more likely to have $\mathrm{HR}+\mathrm{tu}$ mors and $\mathrm{HR}+$ metastases [21]. In a large study with a long follow-up period ( 28 years), $30 \%$ of metastatic breast cancer patients $>70$ years died from non-breast cancer causes [22].

Our study has several limitations. First, it is retrospective and we assume but cannot be certain that experienced clinicians chose to biopsy patients with suspected metastases with a more favorable prognosis. Second, we do not have data on how treatment decisions were affected in patients with discordant biopsy findings. Third, our study was not sufficiently powered to check discordance of $\mathrm{HR}$ and HER2 for different metastatic sites. In one study that examined discordance in 233 distant breast cancer metastases at different sites (76 skin, 63 liver, 43 lung, 44 brain, 7 gastro-intestinal), receptor conversion seemed to occur mostly in liver and brain metastases; however, this was significant only for PR conversion [23].

\section{Conclusions}

Our data confirm the high discordance rate between findings at initial breast cancer diagnosis and findings at metastases. Our findings suggest that patients most likely to benefit from biopsy are those without liver, lung or brain metastases, are younger than 70 years of age, and with missing receptors status at initial diagnosis. Recent American Society of Clinical Oncology (ASCO) guidelines [24] suggest that, in patients with accessible metastases, biopsy for confirmation of metastases and 
Table 4 Effect of biopsy on survival for selected clinical and pathological variables

\begin{tabular}{|c|c|c|c|c|c|c|}
\hline & \multirow[b]{3}{*}{ TOTAL } & \multirow{3}{*}{$\begin{array}{l}\text { No. } \\
\text { Pts. } \\
409\end{array}$} & \multicolumn{2}{|c|}{ All Cause Mortality } & \multirow{3}{*}{$P$ value } & \multirow{3}{*}{ HR $(95 \% \mathrm{Cl})$} \\
\hline & & & No. & $\%$ total & & \\
\hline & & & 331 & 80.9 & & \\
\hline \multicolumn{7}{|l|}{ Biopsy at recurrence } \\
\hline \multirow[t]{2}{*}{ Biopsy } & No Bx & 244 & 222 & 91.0 & & 1.00 \\
\hline & $B x$ & 165 & 109 & 66.1 & 0.000 & $0.64(0.51-0.81)$ \\
\hline \multicolumn{7}{|l|}{ HR \& HER2at initial diagnosis } \\
\hline \multirow[t]{2}{*}{ HR+/HER2+ } & No $B x$ & 38 & 30 & 78.9 & & 1.00 \\
\hline & $\mathrm{Bx}$ & 10 & 6 & 60.0 & 0.499 & $0.74(0.30-1.79)$ \\
\hline \multirow[t]{2}{*}{ HR-/HER2- } & No $B x$ & 35 & 33 & 94.3 & & 1.00 \\
\hline & Bx & 14 & 10 & 71.4 & 0.236 & $0.65(0.32-1.33)$ \\
\hline \multirow[t]{2}{*}{ HR-/HER2+ } & No Bx & 35 & 33 & 94.3 & & 1.00 \\
\hline & Bx & 9 & 5 & 55.6 & 0.562 & $0.75(0.29-1.98)$ \\
\hline \multirow[t]{2}{*}{$\mathrm{HR}+/ \mathrm{HER} 2-$} & No Bx & 99 & 90 & 90.9 & & 1.00 \\
\hline & $\mathrm{Bx}$ & 74 & 49 & 66.2 & 0.097 & $0.74(0.52-1.06)$ \\
\hline \multirow[t]{2}{*}{ HR and/or HER2 missing } & No $B x$ & 37 & 36 & 97.3 & & 1.00 \\
\hline & $\mathrm{Bx}$ & 58 & 39 & 67.2 & 0.001 & $0.45(0.28-0.71)$ \\
\hline \multicolumn{7}{|l|}{ Site of metastasis at recurrence } \\
\hline \multirow[t]{2}{*}{ No liver \& lung or brain metastases } & No Bx & 202 & 182 & 90.1 & & 1.00 \\
\hline & $B x$ & 139 & 86 & 61.9 & 0.001 & $0.63(0.49-0.82)$ \\
\hline \multirow[t]{2}{*}{ Liver \& lung metastases } & No Bx & 17 & 15 & 88.2 & & 1.00 \\
\hline & $\mathrm{Bx}$ & 18 & 17 & 94.4 & 0.539 & $0.80(0.39-1.63)$ \\
\hline \multirow[t]{2}{*}{ Brain metastases } & No Bx & 25 & 25 & 100.0 & & 1.00 \\
\hline & Bx & 8 & 6 & 75.0 & 0.196 & $0.55(0.22-1.36)$ \\
\hline \multicolumn{7}{|l|}{ Age at metastatic recurrence } \\
\hline \multirow[t]{2}{*}{ Age $<70$ years } & No Bx & 207 & 189 & 91.3 & & 1.00 \\
\hline & $\mathrm{Bx}$ & 141 & 92 & 65.2 & 0.000 & $0.62(0.48-0.80)$ \\
\hline \multirow[t]{2}{*}{ Age $>=70$ years } & No Bx & 37 & 33 & 89.2 & & 1.00 \\
\hline & Bx & 24 & 17 & 70.8 & 0.587 & $0.85(0.47-1.54)$ \\
\hline \multicolumn{7}{|l|}{ Stage at initial diagnosis } \\
\hline \multirow[t]{2}{*}{ Stage 0-IIA } & No Bx & 82 & 71 & 86.6 & & 1.00 \\
\hline & Bx & 90 & 56 & 62.2 & 0.025 & $0.67(0.47-0.95)$ \\
\hline \multirow[t]{2}{*}{ Stage IIB-III } & No $B x$ & 161 & 151 & 93.8 & & 1.00 \\
\hline & $\mathrm{Bx}$ & 71 & 51 & 71.8 & 0.034 & $0.71(0.51-0.97)$ \\
\hline
\end{tabular}

$H R$ hormone receptors, HER2 human epidermal growth factor receptor 2, Bx biopsy

retesting of estrogen receptor, progesterone receptor, and HER2 status should be offered. However, evidence is lacking to determine whether modifying anticancer therapy on the basis of a change in receptor status influences clinical outcomes [24]. The ASCO panel consensus was to preferentially use the ER, PR, and HER2 status of the metastasis to direct therapy, if supported by the clinical scenario and the patient's goals. In addition, tissues from metastases may be used to assess molecular differences not limited to receptor level but also to investigate
DNA, RNA, protein and functional pathway levels and to look for actionable mutations that might allow for novel new therapies [5, 25]. Our findings support current recommendations and show that, in selected patients, a biopsy at metastatic recurrence that included HR and HER2 status was associated with significantly reduced mortality.

\section{Abbreviations}

$\mathrm{Cl}$ : Confidence intervals; ER: Estrogen receptor; FISH: Fluorescent in situ hybridization; HER2: Human epidermal growth factor receptor 2; HR: Hazard 
ratio; HR: Hormone receptor; MBC: Metastatic breast cancer; OR: Odds ratio; OS: Overall survival; PR: Progesterone receptor; RHCC: Rambam Health Care Campus

\section{Acknowledgements}

The authors thank Mrs. Myrna Perlmutter for her help in the preparation of this paper. Funding for this service was provided by the Division of Oncology, Rambam Health Care Campus, Haifa, Israel.

\section{Funding}

The project was funded by the Division of Oncology, Rambam Health Care Campus, Haifa, Israel.

\section{Availability of data and materials}

The datasets used and/or analysed during the current study are available from the corresponding author on reasonable request.

\section{Authors' contributions}

SS wrote the manuscript, collected and analyzed the data; TM did the statistical analysis; GF treated the patient and helped to draft the manuscript; KD treated the patient and helped to draft the manuscript; NS did the data collection and analysis; HM helped to draft the manuscript; GBS was the study initiator, helped in the data analysis, and drafted the manuscript. All authors have read and approved the manuscript as submitted.

\section{Competing interests}

The authors declare that they have no competing interests.

\section{Consent for publication}

Not applicable.

\section{Ethics approval and consent to participate}

Page 4 - This study was approved by the ethics committee of Rambam Health Care Campus, Haifa, Israel (Certificate no. 0408-13-RMB); Due to the retrospective nature of this study, consent for participation was not requested.

\section{Author details}

'Division of Oncology, Rambam Health Care Campus, Haifa, Israel. ${ }^{2}$ Statistical Department, Rambam Health Care Campus, Haifa, Israel. ${ }^{3}$ University of North Carolina, Chapel Hill, NC, USA. ${ }^{4}$ Rappaport Faculty of Medicine, Technion-Israel Institute of Technology, Haifa, Israel. ${ }^{5}$ Integrated Oncology and Palliative Care Unit, Rambam Health Care Campus, POB 9602, Haifa 31096, Israel.

Received: 16 December 2015 Accepted: 16 December 2016 Published online: 04 January 2017

\section{References}

1. Siegel RL, Miller KD, Jemal A. Cancer statistics, 2016. CA Cancer J Clin. 2016; 66:7-30. doi:10.3322/caac.21332.

2. NCCN (1AD) NCCN Clinical Practice Guidelines in Oncology (NCCN Guidelines $^{\oplus}$ ) Breast Cancer. Version 12015 1-187. https://www.nccn.org/ professionals/physician_gls/pdf/breast.pdf.

3. Cardoso F, Costa A, Norton L, Senkus E, Aapro M, André F, et al. ESO-ESMO 2nd international consensus guidelines for advanced breast cancer (ABC2). Ann Oncol. 2014:25:1871-87. doi:10.1093/annonc/mdu385.

4. Brennan MJ, Donegan WL, Appleby DE. The variability of estrogen receptors in metastatic breast cancer. Am J Surg. 1979:137:260-2.

5. Criscitiello C, André F, Thompson AM, De Laurentiis M, Esposito A, Gelao L, et al. Biopsy confirmation of metastatic sites in breast cancer patients: clinical impact and future perspectives. Breast Cancer Res. 2014;16:205. doi:10.1186/bcr3630.

6. Aurilio G, Disalvatore D, Pruneri G, Bagnardi V, Viale G, Curigliano G, et al. A metaanalysis of oestrogen receptor, progesterone receptor and human epidermal growth factor receptor 2 discordance between primary breast cancer and metastases. Eur J Cancer. 2014:50:277-89. doi:10.1016/j.ejca.2013.10.004.

7. Pusztai L, Viale G, Kelly CM, Hudis CA. Estrogen and HER-2 receptor discordance between primary breast cancer and metastasis. Oncologist. 2010;15:1164-8. doi:10.1634/theoncologist.2010-0059.

8. Karlsson E, Appelgren J, Solterbeck A, Bergenheim M, Alvariza V, Bergh J. Breast cancer during follow-up and progression - a population based cohort on new cancers and changed biology. Eur J Cancer. 2014;50:291624. doi:10.1016/j.ejca.2014.08.014.

9. Karagöz Özen DS, Ozturk MA, Aydin Ö, Aydin Ö, Turna ZH, Ilvan S, Özgüroglu M. Receptor expression discrepancy between primary and metastatic breast cancer lesions. Oncol Res Treat. 2014;37:622-6. doi:10. 1159/000368312.

10. Curtit E, Nerich V, Mansi L, Chaigneau L, Cals L, Villanueva C, et al. Discordances in estrogen receptor status, progesterone receptor status, and HER2 status between primary breast cancer and metastasis. Oncologist. 2013;18:667-74. doi:10.1634/theoncologist.2012-0350.

11. Katz MH. Multivariable analysis: a practical guide for clinicians. 2nd ed. 2007

12. Qu Q, Zong Y, Fei XC, Chen XS, Xu C, Lou GY, et al. The importance of biopsy in clinically diagnosed metastatic lesions in patients with breast cancer. World J Surg Oncol. 2014;12:93. doi:10.1186/1477-7819-12-93.

13. Thompson AM, Jordan LB, Quinlan P, Anderson E, Skene A, Dewar JA, Breast Recurrence in Tissues Study Group, et al. Prospective comparison of switches in biomarker status between primary and recurrent breast cancer: the Breast Recurrence In Tissues Study (BRITS). Breast Cancer Res. 2010;12: R92. doi:10.1186/bcr2771.

14. Amir E, Miller N, Geddie W, Freedman O, Kassam F, Simmons C, et al. Prospective study evaluating the impact of tissue confirmation of metastatic disease in patients with breast cancer. J Clin Oncol. 2012;30:587-92. doi:10. 1200/JCO.2010.33.5232.

15. Yang YF, Liao YY, Yang M, Peng NF, Xie SR, Xie YF. Discordances in ER, PR and HER2 receptors between primary and recurrent/metastatic lesions and their impact on survival in breast cancer patients. Med Oncol. 2014:31:214. doi:10.1007/s12032-014-0214-2

16. Vignot S, Besse B, André F, Spano JP, Soria JC. Discrepancies between primary tumor and metastasis: a literature review on clinically established biomarkers. Crit Rev Oncol Hematol. 2012;84:301-13. doi:10.1016/j.critrevonc. 2012.05.002.

17. Amir E, Clemons M, Purdie CA, Miller N, Quinlan P, Geddie W, et al. Tissue confirmation of disease recurrence in breast cancer patients: pooled analysis of multi-centre, multi-disciplinary prospective studies. Cancer Treat Rev. 2012:38:708-14. doi:10.1016/j.ctrv.2011.11.006

18. Tobin NP, Harrell JC, Lovrot J, Egyhazi Brage S, Frostvik Stolt M, Carlsson L, TEX Trialists Group, et al. Molecular subtype and tumor characteristics of breast cancer metastases as assessed by gene expression significantly influence patient post-relapse survival. Ann Oncol. 2015;26:81-8. doi:10. 1093/annonc/mdu498.

19. Swain SM, Baselga J, Kim S-B, Ro J, Semiglazov V, Campone M, CLEOPATRA Study Group, et al. Pertuzumab, trastuzumab, and docetaxel in HER2positive metastatic breast cancer. N Engl J Med. 2015;372:724-34. doi:10. 1056/NEJMoa1413513.

20. Slamon DJ, Leyland-Jones B, Shak S, Fuchs H, Paton V, Bajamonde A, et al Use of chemotherapy plus a monoclonal antibody against HER2 for metastatic breast cancer that overexpresses HER2. N Engl J Med. 2001;344 783-92. doi:10.1056/NEJM200103153441101.

21. Diab SG, Elledge RM, Clark GM. Tumor characteristics and clinical outcome of elderly women with breast cancer. J Natl Cancer Inst. 2000;92:550-6.

22. Schairer C, Mink PJ, Carroll L, Devesa SS. Probabilities of death from breast cancer and other causes among female breast cancer patients. J Natl Cancer Inst. 2004:96:1311-21. doi:10.1093/jnci/djh253.

23. Hoefnagel LD, van de Vijver MJ, van Slooten HJ, Wesseling P, Wesseling J, Westenend PJ, et al. Receptor conversion in distant breast cancer metastases. Breast Cancer Res. 2010;12:R75. doi:10.1186/bcr2645.

24. Van Poznak C, Somerfield MR, Bast RC, Cristofanilli M, Goetz MP, GonzalezAngulo AM, et al. Use of biomarkers to guide decisions on systemic therapy for women with metastatic breast cancer: American Society of Clinical Oncology Clinical Practice Guideline. J Clin Oncol. 2015;33:2695-704. doi:10. 1200/JCO.2015.61.1459.

25. Toss A, Cristofanilli M. Molecular characterization and targeted therapeutic approaches in breast cancer. Breast Cancer Res. 2015;17:60. doi:10.1186/ 13058-015-0560-9. 